\title{
IEEE 5G Spectrum Sharing Challenge: A Practical Evaluation of Learning and Feedback
}

\author{
Sreeraj Rajendran, Bertold Van den Bergh, Tom Vermeulen and Sofie Pollin \\ Department ESAT, KU Leuven, Belgium \\ Email: \{sreeraj.rajendran, bertold.vandenbergh, tom.vermeulen and sofie.pollin\}@esat.kuleuven.be
}

\begin{abstract}
We present the results, experiences and takeaways from comparing a diverse set of dynamic spectrum access methods during the IEEE DySPAN 2015 spectrum challenge. Five solutions for coexistence with a given wireless link were implemented and tested in an unknown environment during the conference in Stockholm. The challenge was framed broadly, enabling participants to use their own hardware, antennas, physical layer or medium access control solutions to compete in a unified setup. Each solution was ran two times and ranked using a single metric. Between the two runs the teams were allowed to improve their solution. The metric considered wanted throughput and unwanted interference. In addition to the metric, all solutions were evaluated by a jury. In this paper, we give a detailed overview of the challenge, how we organized it, the participating teams and finally the winners. We conclude with some takeaways on dynamic spectrum access.
\end{abstract}

\section{INTRODUCTION}

It has been nearly two decades since Joseph Mitola coined the term cognitive radio in 1998. A cognitive radio is an intelligent and autonomous system that can adapt its transmission and reception parameters based on the environment. Since then, wireless technology has become a lot more sophisticated. Capacity is boosted by adding more configurable algorithms and by utilizing various spectrum bands. Still it remains an open question how much learning is needed and how relevant it is to tune those knobs and bands dynamically in function of the environment. Learning the environment requires feedback about the environment. This feedback can be obtained by spectrum sensing or from a database. In essence, the main questions related to the design of dynamic spectrum access radios, or cognitive radios as Mitola framed them, are (a) how much benefits can be achieved by learning and adapting, compared to other solutions at physical or medium access layer, and (b) what feedback information should be available to facilitate learning and adaptation.

To start the debate towards answering these questions, IEEE DySPAN 2015 has organized a spectrum challenge ${ }^{1}$, which was designed in such a way that any wireless research group could participate. It was up to the teams to decide how much effort they would spend on antenna design, novel hardware, physical layer solutions or medium access protocols. Each team could individually decide how much adaptation and learning to include. As a novel enhancement to the learning process, a database was implemented with real-time feedback

\footnotetext{
${ }^{1} \mathrm{http}: / /$ dyspan2015.ieee-dyspan.org/content/5g-spectrum-sharingchallenge
}

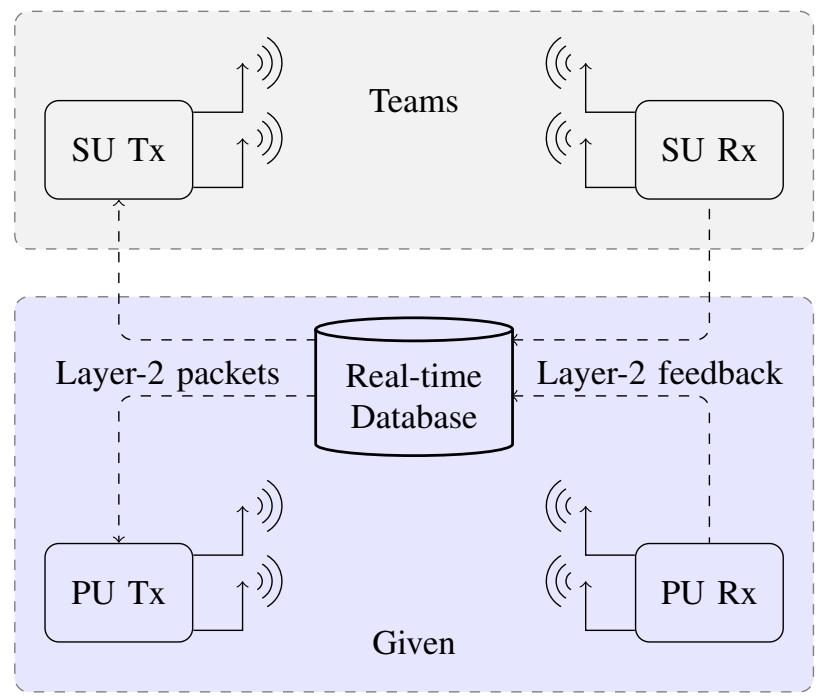

Fig. 1: Spectrum challenge setup showing the real-time feedback from the database.

about the throughput of the incumbent link. This would give each team instantaneous feedback about interference caused to the primary receiver, enabling detailed learning of the optimal transmission parameters.

While receiver feedback is currently not present in any spectrum sharing database, it is not unrealistic to explore. First, it would give an upper bound on what learning and feedback would bring. Second, we see a trend towards more and more receiver based regulation, giving the receiver a larger role in the spectrum optimization problem ${ }^{2}$. Third, a lot of wireless systems already implement some kind of acknowledgment, which is receiver feedback that can be used to optimize transmitter parameters.

A high level overview of the IEEE DySPAN 2015 challenge setup is given in Figure 1. The database provides packets and performance metrics in real-time to the transmitter and collects statistics about the system performance. Both the primary user (PU) and the secondary user (SU) radio were connected to the database and could at most use two antennas. The PU system was based on IEEE 802.15.4, which was chosen as this is one of the cheapest wireless system one can find. Therefore, even on a very limited budget, each team would be able to setup the PU system in their lab. For the challenge, the PU system was implemented using a software defined radio

\footnotetext{
${ }^{2}$ www.whitehouse.gov/sites/default/files/microsites/ostp/pcast_ spectrum_report_final_july_20_2012.pdf
} 
(SDR) IEEE 802.15.4 implementation running on two USRPs. This implementation follows the standard but has different RF properties compared to the cheap dongle. The main idea being that while some properties are given, some remain to be learned and calibrated by using the feedback in the database or spectrum sensing.

To understand the current state of these spectrum sharing algorithms, various competitions were held in the past. The DARPA spectrum challenge ${ }^{3}$, a prominent one among them, put state of the art (SoA) cognitive algorithms to the test by designing competitive and cooperative competitions. This challenge gave insight to the adaptability of the SoA radio algorithms in using the spectrum aggressively or sharing it among other users based on the environment. The difference with the IEEE 5G spectrum sharing challenge is that the DARPA challenge focused mainly on cooperation using a given radio and physical layer challenges and not so much on the learning or feedback information requirements of a cognitive radio network. Whereas the IEEE 5G spectrum sharing challenge focused on learning and feedback information requirements of a cognitive radio network using database aided spectrum sharing. The participants were given complete freedom in their radio and physical layer design, within the spectrum constraints. Another spectrum sharing challenge is the SPECTRUM-SHARC Student Cognitive Radio Contest ${ }^{4}$, where students are given access to the CORNET cognitive radio testbed. Here the hardware was fixed but teams were allowed to make waveform changes. The goals of the challenge are similar to the $5 \mathrm{G}$ spectrum sharing challenge in that the $\mathrm{SU}$ needs to maximize its throughput while minimizing its interference to the PU. However, realtime feedback of the PU and SU throughput and physical design freedom made IEEE $5 \mathrm{G}$ spectrum sharing challenge different from SPECTRUMSHARC challenge.

The rest of the paper is organized as follows. Section II defines the challenge setup and the winning parameters. A brief overview of participating teams and their radio designs are presented in Section III. Section IV details the actual challenge results and discusses shortcomings of the used methods. Takeaways and conclusions from the challenge are presented in Section V and VI respectively.

\section{Challenge Implementation}

The challenge was designed to meet a range of criteria: enable breadth in the solutions, enable teams with no hardware or expertise to participate and enable learning with real-time feedback about the primary user throughput statistics. Given those high-level criteria, a standard IEEE 802.15.4 stack for a widely available wireless dongle was provided to ensure the participation of teams with only MAC experts who want to avoid physical layer implementation hassles. The heart of the solution was a database, that could in real-time talk to the dongle's IEEE 802.15.4 standard stack, as well as GNU Radio and LabVIEW software defined radio systems. As a result, PU and SU could be any system from a cheap dongle

\footnotetext{
${ }^{3}$ www.darpa.mil/program/spectrum-challenge

${ }^{4} \mathrm{http}: / /$ radiocontest.wireless.vt.edu/index.html
}

with an IEEE 802.15.4 stack to custom implementations on SDRs.

To make the competition more challenging, the PU simultaneously transmitted on four predefined frequency bands with channel spacing of $5 \mathrm{MHz}$. The secondary user had to maximize its throughput over the same $20 \mathrm{MHz}$ which the PU was using. The center frequency of the band is $2.3 \mathrm{GHz}$, which was a dedicated interference-free band used for the challenge. As a result, teams had to learn only the room characteristics (fading and pathloss parameters), PU traffic patterns and the exact PU RF properties of the USRP-X310s used.

\section{A. Primary User Setup}

The PU radio had a four channel GNU Radio based IEEE 802.15.4 stack [1] with an O-QPSK physical layer connected to a USRP front-end via Ethernet interface as shown in Figure 2. The PU used four independent streams which were configured to transmit packets independently on the four channels using a fixed packet length of 127 bytes. The packet generator block controlled these four streams by requesting data from the database, re-sizing it and pushing the packets to the independent 802.15.4 MAC modules of the streams. The timing of these packets was controlled by the packet generator and was based on four instantiations of the same random distribution. The packet generator switched to a new distribution every 3 minutes to mimic the non-stationary nature of channel occupancy.

The random distributions used in the challenge consisted of

- a uniform distribution with minimum and maximum interpacket duration of $8 \mathrm{~ms}$ and $150 \mathrm{~ms}$ respectively;

- two poisson distributions with means $20 \mathrm{~ms}$ and $150 \mathrm{~ms}$;

- a back-to-back transmission scheme with minimal interpacket period of $5 \mathrm{~ms}$.

All the parameters of these distributions were configured before the start of the challenge. A common starting seed was used for all distributions which helped in keeping the randomness fixed across the teams during both phases of the challenge. The implemented code can be easily adapted to follow different distributions to test more complex channel occupancy scenarios in future. In reality, there can be many different spectrum occupancy scenarios, so a fairly large set of possible random distributions were chosen for the challenge.

\section{B. Database and Feedback}

Giving feedback of the PU performance to the SU radio should allow for much faster and more fine grained adaptation of the SU settings. This feedback mechanism was implemented using a central database server. The server delivers random packets to both the PU and SU. Received packets are delivered back to the database server where they were verified and statistics were updated. These statistics were accessible from both PU and SU.

To generate packets with strong randomness at high throughput AES-128 was used in counter mode with a random key. It is important that frames are sufficiently random since otherwise they could be compressed before transmission. A 


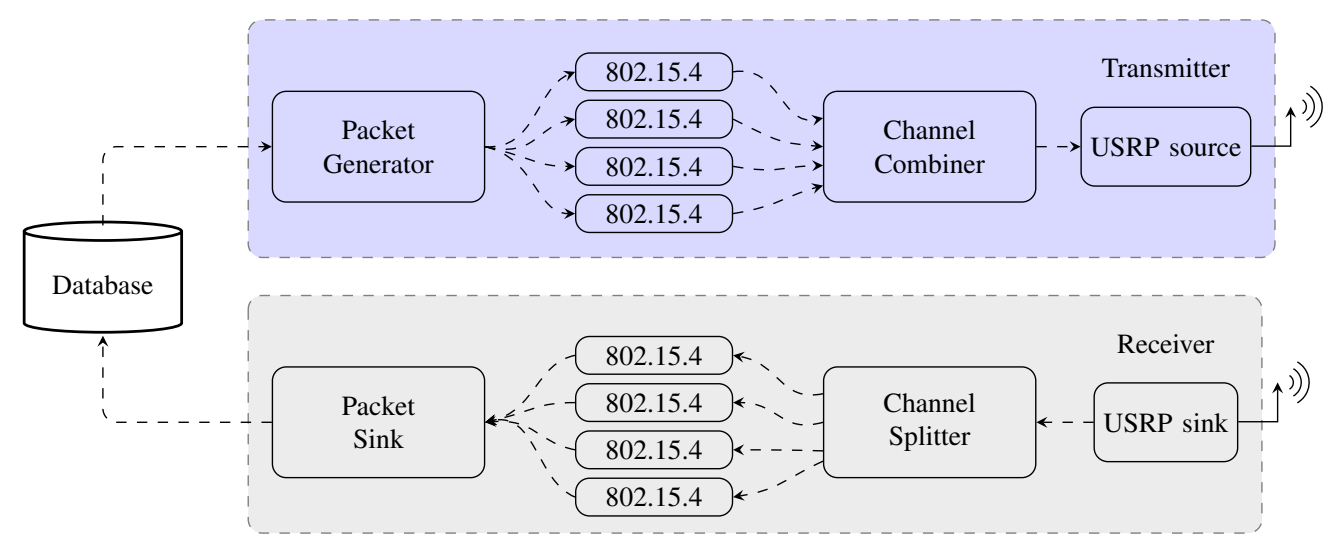

Fig. 2: Primary user setup

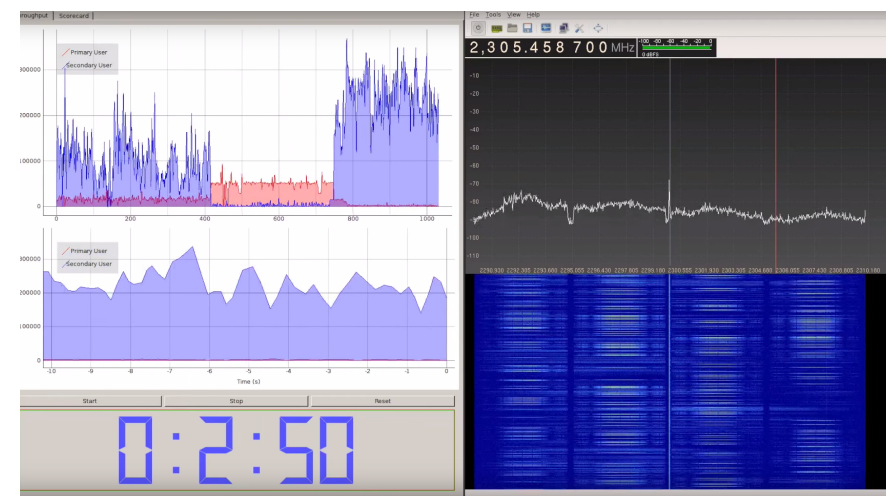

Fig. 3: Challenge visualization: Instantaneous PU and SU throughput (left) with live FFT (top-right) and waterfall plots (bottom-right).

secure message authentication code (MAC) is calculated over the frame to detect if it has been corrupted. A sequence number is added to prevent packets from being counted multiple times.

Since we feel that this database platform coupled with client libraries for both GNU Radio and LabVIEW may be useful to others it is made publicly available ${ }^{5,6}$. The PU implementation used in the challenge is also made available in this package.

\section{Challenge visualization}

A challenge visualization was setup for live monitoring and analysis of the status of challenge. A screen capture of the used visualization is shown in Figure 3. In the left column, graphs showing instantaneous $\mathrm{PU}$ and $\mathrm{SU}$ throughput are plotted along with the elapsed time. A live FFT and waterfall plot, as shown in the right column of the figure, was used for monitoring the channel occupancy patterns of both pairs of radios. A custom visualization module connected to the real-time database was used for displaying the PU and SU performance. A software defined radio receiver powered by the GNU Radio and the Qt graphical toolkit $\mathrm{Gqrx}^{7}$ was used for displaying spectrum details.

\footnotetext{
${ }^{5}$ http://claws.be/spectrum-challenge/

${ }^{6} \mathrm{https}: / /$ github.com/networkedsystems/dyspanchallenge_2015

${ }^{7}$ http://gqrx.dk/
}

\section{Challenge Phases}

The challenge consisted of two phases: a learning phase and a test phase as given below.

- Learning Phase (10 min)

- SU can learn PU statistics;

- PU feedback is provided to optimize the SU parameters;

- No scoring in this phase.

- Test Phase (10 min)

- Actual scoring phase;

- PU transmission statistics same as during the learning phase;

- SU penalized for interference.

Each phase had a duration of 10 minutes. The SU radio could learn about the environment, the PU transmission statistics and the exact PU transmitter and receiver RF properties impacting the interference sensitivity. This acquired knowledge could then be used to calibrate the SU parameters and algorithms to improve its performance. During the learning phase, the SU could also test its algorithms and validate them efficiently by making use of the real-time feedback from the database. The final scores were only calculated during the test phase of the challenge.

\section{E. Challenge metric}

Two winners were selected. One was selected based on a single metric combining both SU and PU throughput, which could be measured unambiguously from the database. The final challenge score was represented by the product of $\mathrm{SU}$ throughput $\left(T_{\mathrm{SU}}\right)$ and $\mathrm{PU}$ satisfaction. The PU satisfaction $\left(S_{\mathrm{PU}}\right)$ was calculated from the offered PU throughput $\left(\widehat{T}_{\mathrm{PU}}\right)$ and the delivered PU throughput $\left(T_{\mathrm{PU}}\right)$ as given in Equation 1. A maximum throughput loss tolerance of $10 \%$ was allowed. More than $10 \%$ PU throughput loss would be counted as zero PU satisfaction.

$$
\begin{aligned}
\text { Score } & =T_{\mathrm{SU}} \times S_{\mathrm{PU}} \\
S_{\mathrm{PU}} & =\max \left(0, \frac{10}{9} T_{\mathrm{PU}}-\widehat{T}_{\mathrm{PU}}\right) .
\end{aligned}
$$

In addition to this single performance metric, which can be measured but does not capture the novelty of the solution, a 
second winner that was selected by a jury was announced. The jury evaluated mainly the maturity of the solution, and most importantly the breadth, i.e., how much cognitive aspects were considered in the final solution. This would exclude nonadaptive teams, or teams failing to take advantage one way or another from the PU or SU feedback enabled in the system.

\section{SECONDARY USER AlgorithmS}

The challenge was designed to enable a large variety of teams to participate in the event. Eventually, 5 teams were selected to compete, using techniques ranging from special waveforms to advanced statistical PU profiling techniques. A brief overview of each team's implementation is given below. The summary of the used physical layer parameters and coexistence techniques can be found in Table I. Each team published the details of their solution as an IEEE DySPAN 2015 challenge paper [2]-[6].

\section{A. Team 1: KIT}

The team from Karlsruhe Institute of Technology (KIT) used a cross layer optimized secondary user system [2] for the challenge. They employed a secondary user waveform with high spectral efficiency, sensing based on energy detection with thresholds that are learned during the learning phase of the challenge and diversity gain which is achieved using multiple antennas. The channel occupancy knowledge is accommodated in the MAC layer of the SU system. The SU transmitter synchronously sensed all the four channels. The measured power levels are then used to adapt the energy detection threshold and one packet is transmitted on every detected free channel. Static learning is employed in the learning phase of the challenge to learn about the channel utilization probabilities and reinforcement learning is used in the test phase of the challenge.

\section{B. Team 2: CNCT/TUI}

A joint team from CONNECT/CTVR, Trinity College Dublin and Technische Universität Ilmenau (TUI) devised a system that makes use of a state machine consisting of sensing, learning, decision making and transmit/receive [3]. A four channel frequency domain energy detection is used during the sensing state. The best modulation and coding schemes are learned during the learning phase of the state machine. The PU channel occupancy parameters are also sensed and a channel distribution is built with the sensed information. This is used to model the PU pattern as a Markov chain which is used for SU transmission channel selection.

\section{Team 3: AIT}

The team from Athens Information Technology (AIT) used parasitic directional antennas and tried to transmit in the blind spots of the primary user receiver [4]. The system selects the best beam pattern from a set of patterns which is learned during the learning phase of the challenge. The system employs adaptive power control based on PU throughput feedback from the database.

\section{Team 4: FORTH-ICS}

The team from Foundation for Research and TechnologyHellas (FORTH) used a custom developed PCIe SDR device with a standard $802.11 \mathrm{~g}$ stack [5]. The available $20 \mathrm{MHz}$ spectrum is virtualized and the system can transmit in four adjacent $5 \mathrm{MHz}$ channels in parallel. During the learning phase the SU discovers available SNR on each channel. Furthermore, the maximum transmission power which will not trigger the PU CCA mechanism is measured. The PU inter frame time period, frame size distributions and the PU channel switching time are learned during the learning phase of the challenge by monitoring and decoding PU transmissions. The transmission power, minimum packet size and frame distributions thus learned are used in the test phase of the challenge.

\section{E. Team 5: FR}

A group from San Diego State University and University of California San Diego designed a SU radio waveform to overlay the PU channels without employing any monitoring or learning [6]. The designed waveform creates negligible interference to the PU waveform avoiding the need for any learning about the primary user statistics.

\section{Challenge Results and Discussion}

Two runs (20 min for each team per run) of the challenge were conducted and the winner was selected based on the best scores out of two runs. The number of runs were fixed to two in order to give the participants a second opportunity to fine tune their algorithms. The availability of dedicated spectrum and the reproducible PU spectral occupancy pattern made the performance results repeatable. Figure $4 \mathrm{a}$ and Figure $4 \mathrm{~b}$ details the SU and PU performances respectively. Figure $4 \mathrm{a}$ summarizes the bytes transferred by each team during both runs of the challenge. Even though most of the teams were able to achieve high SU throughput, only two teams managed to keep the PU throughput loss below 10\% (Figure 4b). Some teams tried to overcome this using short length packets and fine transmit power control while others used directional systems to keep the interference level low.

\section{A. Winners}

From the final scores computed from both runs, team CNCT/TUI was selected as the winner based on the metric. CNCT/TUI's high throughput PHY-MAC combination along with beamforming techniques helped in achieving the winning score. Team KIT was selected by the jury as the winner based on the breadth and consistency of the solution. Team KIT performed consistently well with their spectrally efficient PHY and short length packets. Even though team AIT employed many features including adaptive power control based on the PU feedback, they failed in suppressing the carrier leakage from their radio front-end which interfered continuously with the PU. Team FORTH also had difficulties in controlling the interference with the PU as their algorithm assumed only a single channel usage by PU at a time. The non-interfering signal design from team FR also performed badly as the 


\begin{tabular}{lll}
\hline Team & Physical Layer & Coexistence Techniques \\
\hline \multirow{4}{*}{ KIT } & $\begin{array}{l}\text { Fully parameterizable Filter Bank Multicarrier (FBMC) PHY } \\
\text { 256 Subcarriers over 4 channels with 5 MHz each, 2 b/s/Hz } \\
\text { Low adjacent channel interference } \\
\text { High spectral efficiency } \\
\text { Short packets for low probability of interference }\end{array}$ & $\begin{array}{l}\text { Adaptive noise floor estimation, no calibration needed } \\
\text { Energy detection gives reliable information about PU beyond database } \\
\text { Past knowledge from SU transmitter sensing used to improve detection probability } \\
\text { Locally sensed busy channels are temporarily blacklisted } \\
\text { Omnidirectional antennas ensure consistent performance in dynamic scenarios }\end{array}$ \\
\hline \multirow{2}{*}{ CNCT/TUI } & $\begin{array}{l}\text { OFDM PHY } \\
\text { Receive on 4x5MHz channels at Rx } \\
\text { Directional Antennas }\end{array}$ & $\begin{array}{l}\text { Efficient multi-threaded solution for simultaneous multi-channel transmission } \\
\text { Noise floor estimation on each channel which improves PU detection performance } \\
\text { Best modulation and coding scheme selected during the learning phase } \\
\text { PU channel occupancy modeled as a Markov chain } \\
\text { SU's transmission channel selected based on this Markov model }\end{array}$ \\
\hline \multirow{2}{*}{ AIT } & $\begin{array}{l}\text { Tx-Rx directional Yagi-Uda planar parasitic antennas } \\
\text { OFDM Modulation with QPSK or 16QAM }\end{array}$ & $\begin{array}{l}\text { Modulation selected based on PU and the SU throughput feedback } \\
\text { SU transmits between two adjacent PU channels to avoid interference } \\
\text { (e.g 2.295 - 2.3 GHz) } \\
\text { Dynamic gain control to minimize PU interference and boost SU's throughput }\end{array}$ \\
\hline \multirow{2}{*}{ FORTH-ICS } & $\begin{array}{l}\text { 4x IEEE 802.11a/g @ 5 MHz (SU Tx/Rx) } \\
\text { 4x IEEE 802.15.4 (PU detector) } \\
\text { Abstraction in OS - everything appears as typical interface }\end{array}$ & $\begin{array}{l}\text { Virtualization in time and frequency domain } \\
\text { PU frame, inter-frame and channel transition durations are decoded } \\
\text { Decoded parameters used to select packet length and modulation parameters }\end{array}$ \\
\hline \multirow{2}{*}{ FR } & $\begin{array}{l}\text { Custom physical layer waveform } \\
\text { FSK modulated tones used as the base waveforms }\end{array}$ & $\begin{array}{l}\text { SU waveform exhibits negligible projection on the PU waveforms } \\
\text { No mutual interaction between PU and SU waveforms } \\
\text { Transmit gain control based on the feedback }\end{array}$ \\
\hline
\end{tabular}

TABLE I: Secondary user features

implemented adaptive power control mechanism failed in reducing the interference.

Referring to the feature list we could conclude that a radio with (a) local sensing based learning, (b) spectrally efficient physical layer and (c) directional antennas or equivalent adaptive power control for reducing interference are promising building blocks for dynamic and cognitive spectrum access. The implementation challenges faced by the teams and feedback from each team after the challenge is summarized in Table II. Even though the participating radios were not fully context aware, the challenge showed that promising technological building blocks to design dynamic spectrum access (DSA) solutions for challenging PU systems exist.

\section{TAKEAWAYS}

\section{A. General discussion}

Given the breadth of all possible solutions for realizing cognitive radio, the challenge entries explored several technologies for DSA. Teams designed their solution, sometimes focusing on a single technique (e.g., FR) or by combining many known techniques (e.g., CNCT/TUI). Although it is difficult to draw general conclusions from the results of the challenge, the following observations are worth discussing:

- Given the metric, it made sense to go for high throughput designs to make a chance to win based on the metric. Yet, teams failed to sufficiently focus also on interference avoidance to improve the PU satisfaction measure.

- Directional antennas were advantageous during the challenge, as the setup was static and hence it was very easy to point to the SU receiver, and away from the PU receiver. Some teams achieved this by manual configuration, others learned it during the learning phase.

- Due to the dynamic nature of cognitive radio for spectrum sharing hard-coded assumptions about the PU can be dangerous. E.g., FORTH-ICS had a brilliant solution that

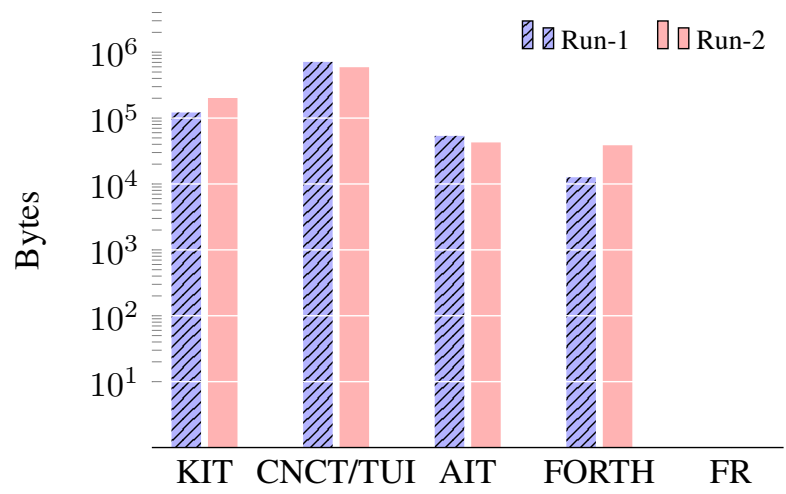

(a) SU Bytes transfered

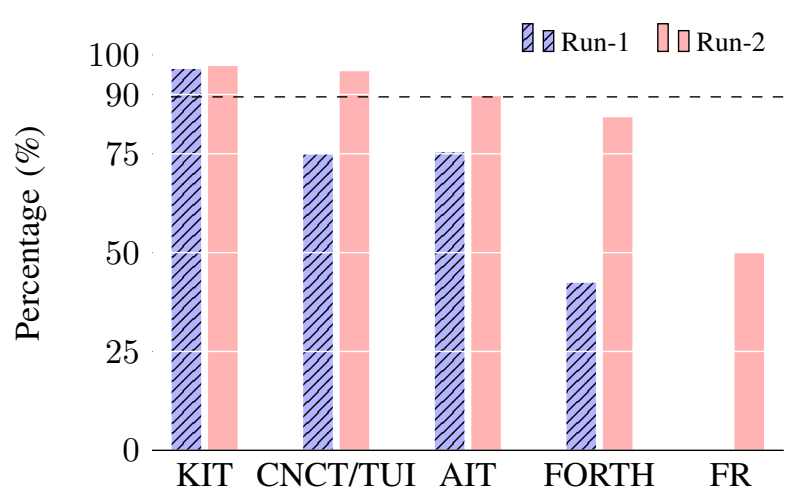

(b) PU packet success percentage

Fig. 4: Primary and Secondary user performance

however assumed that the PU only used a single channel at a time. It was difficult to adapt the solution on the spot.

- While all solutions had some form of intelligence and adaptation (minimally power control), most teams failed to take advantage of the learning phase to really improve the system. A main reason for this is that the PU was 


\begin{tabular}{ll}
\hline Team & Challenges and Takeaways \\
\hline KIT & $\begin{array}{l}\text { (-)Non-deterministic latency in PC host based SDR is a serious issue, but can be mitigated by moving control algorithms to FPGA. } \\
\text { (+)Adaptive modulation and code rate along power control are key required features } \\
(+) \text { Short packets can easily reduce interference }\end{array}$ \\
\hline CNCT/TUI & $\begin{array}{l}\text { (+)Exploiting directional antennas really improves DSA performance } \\
(+) \text { Moving the host code close to the radio helps to solve SDR latency issues } \\
(+) \text { Noise floor calibration with simple energy detection works in practice }\end{array}$ \\
\hline AIT & $\begin{array}{l}\text { (+)Beamforming techniques using parasitic antennas greatly improve PU-SU isolation } \\
(+) \text { Transmitting in the PU spectrum nulls is effective with proper power control } \\
(-) \text { RF frontend calibration is a must, carrier leakages can result in high interference }\end{array}$ \\
\hline FORTH-ICS & $\begin{array}{l}\text { (-)Non-practical assumptions about PU statistics can harm the entire system } \\
(-) \text { Forced to use small constellations(BPSK) as the interference with PU was high as a result of wrong assumptions } \\
(+) \text { SDR virtualization over existing standards can be used with proper adaptations [5] }\end{array}$ \\
\hline FR & $\begin{array}{l}\text { (+)Knowledge about the PU physical layer can be used to design interference free waveform } \\
(+) \text { Automatic transmit power control is essential even with interference free waveform design } \\
(+) \text { Complex PU statistics learning can be avoided with these designs } \\
(-) \text { SU transmit power should be tightly controlled using PU feedback which can otherwise result in high interference }\end{array}$ \\
\hline
\end{tabular}

TABLE II: Team feedback

very dynamic and learning this was of course very challenging. While gains by automated learning were not logged, we did log gains by human intervention in between the two runs. Indeed, all the teams did better the second time, which proves that by some human or manual calibration, all designs could be improved. The ideal cognitive radio should however be able to learn it's configuration autonomously, or ideally even outperform human calibration. We are still far from that reality but some promising building blocks to enable this were tested during the challenge.

- While there was real-time feedback of PU interference, one would expect that it should be easy to design a scheme that meets the interference constraints, by simply disabling the transmitter when the PU interference ratio became too high. Most teams did not strongly rely on this feedback and hence did not manage to keep interference below the target levels.

\section{B. Suggestions for future work}

The challenge results revealed that solutions utilizing time domain spectral vacancies yielded good results. The SoA research also suggests for devising policies which make use of spectral vacancies $[7,8]$. The main challenges in realizing such a system include limitations in observing the channel in a half duplex radio receiver, modeling limitations of the non-stationary PU transmissions [7] and the inherent nondeterminism of channel occupancy. The solutions presented during the challenge tried to tackle some of these challenges, for example predicting the PU channel occupancy pattern using a hidden Markov model (HMM) [3]. However, models based on such dynamic Bayesian Networks (DBNs) like Kalman filters and HMMs may be sub-optimal mainly due to relatively simple state transition structures or internal state space structure. Models employing deep learning techniques which have rich internal state representations and flexible nonlinear functions are shown to outperform these conventional techniques. DSA systems employing such models are yet to be investigated. An in-band full duplex system can also benefit DSA as it improves the quality of observation of the environment which aids instantaneous response, for example a SU can stop an ongoing transmission if it detects a PU transmission in the same channel.

The database feedback was used only by a few teams during the learning phase to test the robustness of their sensing schemes or for transmitter power control. While most of the challenge entries relied on local sensing and directional antennas for improving the metric, the use and value of feedback from the PU is not thoroughly investigated. Such an investigation makes sense as the feedback helps in controlling SU interference in mission critical PU scenarios. It has been shown that reinforcement learning techniques like Q-learning which makes use of PU feedback can improve the system performance [9]. On the other hand, the practicality of enabling feedback and deciding the type of feedback should be debated more before reaching a proper consensus. For example, if the SU can decode the PU transmissions it may be possible to infer the extent of interference caused. Alternatively, the PU could provide this information over a side-channel, e.g. the internet. In the future, it would also be interesting to continue building on the initial setup by adding more challenges that focus more on the metric like the PU latency, the scenario, the PU traffic patterns, the PU RF properties, the learning phase and availability of detailed feedback.

\section{CONCLUSION}

Spectrum is scarce, and to make optimal use of it we will have to share it. Sharing needs adaptive techniques that enable radios to adapt their configurations to the exact properties of the interfered system: packet transmission parameters as well as detailed RF properties and sensitivities. In the IEEE DySPAN 2015 challenge, a system was implemented giving the competing users optimal feedback about their impact on the legacy, primary system. Surprisingly, it was learned that keeping interference below well communicated limits was still a major challenge. The reasons for this were very broad, from hardware non-idealities that were underestimated, to failure 
to take advantage of the exact feedback. Nevertheless, two teams achieved a very high throughput showing that with existing technology it is possible to design a DSA system in practice, even for a very challenging and dynamic primary user scenario. This was however enabled by some human interventions and algorithm changes in between the two runs. Even though we understand the key features required, the main challenge remains in adding full context awareness in radios that can adapt without much human intervention.

As a main conclusion for the IEEE DySPAN 2015 challenge, we can say that participating teams learned a lot, from the challenge and from each other, and went home with an improved design and solution. The design and organization of such small, targeted challenges, can hence serve the community tremendously, as it enables to benchmark solutions, compare them, learn from each other, and give teams prime opportunities to encounter challenges that they had not yet considered when testing in the lab only. While it is hard to generalize the winning solutions as the best or must have DSA technology, it is possible to generalize some lessons learned about learning, adaptability, human intervention and how challenging wireless communication really is. Empirical results show that effective time domain utilization of spectral vacancies yielded good results which is also backed by SoA research. Policies making use of time domain sharing along with viable feedback schemes for PU interference reduction should be more investigated for enabling DSA systems in practice.

\section{ACKNOWLEDGEMENT}

The authors would like to thank the anonymous reviewers for their valuable comments and suggestions to improve the quality of the paper. They are also grateful to National Instruments for the hardware support during the challenge and for the final prizes. Finally the authors would like to thank all challenge participants for their enthusiasm and co-operation.

\section{REFERENCES}

[1] B. Bloessl, C. Leitner, F. Dressler and C. Sommer, "A GNU Radio-based IEEE 802.15.4 Testbed," Proceedings of 12. GI/ITG KuVS Fachgespräch Drahtlose Sensornetze (FGSN 2013), Cottbus, Germany, September 2013, pp. 37-40.

[2] A. Kaushik, F. Wunsch, A. Sagainov, N. Cuervo, J. Demel, S. Koslowski, H. Jäkel and F. Jondral, "Spectrum sharing for $5 \mathrm{G}$ wireless systems (Spectrum sharing challenge)," Dynamic Spectrum Access Networks (DySPAN), 2015 IEEE International Symposium on, Stockholm, 2015, pp. 1-2.

[3] J. Tallon, C. Blumm, A. Puschmann, F. Paisana, J. van de Belt, P. di Francesco, N. Kaminski and H. Ahmadi, "Coexistence through adaptive sensing and Markov chains," Dynamic Spectrum Access Networks (DySPAN), 2015 IEEE International Symposium on, Stockholm, 2015, pp. 7-8.

[4] D. Ntaikos, K. Ntougias, B. Gizas, F. Verdou and C. B. Papadias, "Low-complexity air-interface-agnostic cooperative parasitic multiantenna spectrum sharing system," Dynamic Spectrum Access Networks (DySPAN), 2015 IEEE International Symposium on, Stockholm, 2015, pp. 5-6.

[5] S. Papadakis, M. Surligas, A. Makrogiannakis, K. Triantafyllakis, G. Vardakis, A. Tzougarakis and G. Floros, "Robust spectrum sharing through virtualization," Dynamic Spectrum Access Networks (DySPAN), 2015 IEEE International Symposium on, Stockholm, 2015, pp. 9-10.

[6] F. Harris, R. Bell and V. K. Adsumilli, "Spectrum sharing between a ZigBee frequency hopper and an FSK modem," Dynamic Spectrum Access Networks (DySPAN), 2015 IEEE International Symposium on, Stockholm, 2015, pp. 3-4.
[7] Y. Liu and A. Tewfik,,"Primary Traffic Characterization and Secondary Transmissions," in IEEE Transactions on Wireless Communications, vol. 13, no. 6, June 2014, pp. 3003-3016.

[8] S. Huang, X. Liu and Z. Ding, "Opportunistic Spectrum Access in Cognitive Radio Networks," INFOCOM 2008. The 27th Conference on Computer Communications. IEEE, Phoenix, AZ, 2008, pp. 2101-2109.

[9] K. A. Yau, P. Komisarczuk and P. D. Teal, "Reinforcement learning for context awareness and intelligence in wireless networks: Review, new features and open issues" in Journal of Network and Computer Applications, vol. 35, no. 1, January 2012, pp. 253-267. 